\title{
Study on College Students' Learning Burnout
}

\author{
Weidong $\mathrm{Wu}$ \\ Department of Education, Dezhou University \\ Dezhou 253023, Shandong, China \\ E-mail: wuweidong0911@eyou.com
}

\begin{abstract}
Learning burnout is students' passive mental state to the learning. College students' learning burnout is closely related with the bad style and culture of the society, the employment pressure, the deficiency of college education and college students' own mental factor. Therefore, to overcome the learning burnout, college and teachers must correct the deficiencies in the education and teaching, and students should also change themselves actively.
\end{abstract}

Keywords: College students, Learning burnout, Causes, Countermeasures

\section{Definition of learning burnout}

When students have no interests or lack in enthusiasms for the learning but can not but do it, they will be sick of the learning, and feel tired in body and mind, that state is called as the learning burnout (Yang, 2005, P.54-58). Generally, the learning burnout has following characteristics. (1) Exhausted emotion. For this characteristic, students generally lack in enthusiasms in their learning, and they feel exhausted, nervous, and frustrated, and they can not centralize their attentions on the learning. Students' emotion is in the extreme tiredness, and the emotional resource is exhausted, and the learning enthusiasm is very low. (2) Lacking in humanization. That means students often treat others with the bland, passive, and negative attitude, and they can not trust others, and they are unbelieving and overcritical to others. The attack behavior is an extreme representation of the learning burnout. On the one hand, students' attack behaviors increase, and their human frictions increase. On the other hand, they will aim at themselves and harm themselves. (3) Low achievability. Learning burnout will induce low achievability. Students will undervalue their achievements, and some of them will even negate what they did.

\section{Causes of college students' learning burnout}

In recent years, many surveys about college students showed that the phenomenon of learning burnout in college students was serious. For example, Zhang Shu et al's survey about 438 college students in Inner Mongolia Municipality indicated that college students' level of learning burnout was higher (Zhang, 2009, P.140-142); Li Weiwei et al's survey about 340 college students also showed that quiet part of colleges students had learning burnout (Li, 2007, P.19-21). College students assume the burden of national building, and the existence of learning burnout would seriously influence the learning and the enhancement of learning efficiency, which goes against the accomplishment of the teaching task of college and the implementation of the educational target, and blocks college students' healthy development in body and mind at the same time. College students' learning burnout mainly comes from following causes.

\subsection{Influence of bad social style and culture}

Bad social style and culture are the important exterior causes of college students' learning burnout. College is not a closed place all along, and the opening characteristic of college students' idea also makes the social style and culture to continually impact the campus culture and college students' consciousness. China is in the transformation term of the society, and various passive ideas and concepts are strongly impacting college students' immature mentality. The practicality, the money worship and the gastronomy are seriously corroding college students' heart, and many college students have been eager for quick success and instant benefit, and they feel that it is too far to change their economic state by the learning, so they will exclude and tire in the learning.

\subsection{Unprecedented employment pressure}

With the popularization of higher education, college students assume unprecedented employment pressure. In 2008 , the 
amount of college graduates in China achieved 5.6 million, and this number achieved 6.1 million in 2009, and this number will achieve 6.3 million in 2010. With unemployed graduated in past years, college students will be large employment group every year. But the actuality that the supply exceeds the demand will make them very difficult to find an ideal work, and even they can be employed, but the salary will be less than peasant workers, so the idea of useless learning will be grew crazily, and they will early want to find a good job, not study hard.

\subsection{Deficiencies in college education}

One abuse of the higher education in China is to emphasize the theory but ignore the practice. The course setting has not emphasized the actual demand of the society and college students, but too much theoretical courses, without practical courses. In teachers' teaching, they also only pay attention to the teaching of theoretical knowledge, but ignore the practices, so many students have low cognition to their learned knowledge. One survey showed that only $5.52 \%$ of college students thought that knowledge was useful, but $50.68 \%$ of them thought that knowledge was useless (Huang, 2007, P.54). Students thought that the learned knowledge has not practical value, which makes them to exclude the courses and knowledge what they learned to some extent, and have low identification to their specialty. Teachers' teaching level also impacts students' learning, and with the enrollment expansion of colleges, the amount of teacher expands quickly, but the total quality of teacher has not been enhanced accordingly, and many students don't satisfy teachers' occupational morality, individual culture, teaching method, and teaching level, which also make them to produce the learning burnout to some extent.

\subsection{Causes in college students}

College students' learning burnout is closely related with their self-efficiency feeling and self-attribution. Some college students had depended on family and teachers too much for a long term, with bad independence of learning, and they could not adapt the learning mode in colleges and they failed in learning, so their self-efficiency feeling would be largely reduced. When facing learning failure, the attribution tendency is not right too. They always attributed their failures to their low ability, so their enthusiasm of learning will be largely harmed, and they will feel helpless in study. Some students also lack in the ability to delay satisfaction. They have not far eyesight, and they only pay attention to the immediate interests, and they can not control themselves in learning, which is one of subjective cause of learning burnout.

\section{Countermeasures of college students' learning burnout}

\subsection{Colleges should satisfy students' demand for practice and emphasize students' psychological health}

Colleges should really emphasize the practice teaching, and increase the hardware investments of the practice teaching, and perfect many establishments such as lab, computer lab, and multimedia to fulfill students demand for practices. In addition, colleges should set up practice courses for students' future employment and development, and correct the mode emphasizing the theoretical courses. At the same time, college should also emphasize college students' education of psychological health. Loving learning and being happy in learning are one standard to measure college students' psychological health. Colleges should further correct students' wrong learning cognition, improve their learning method, strengthen their successful experience, enhance their learning motivation, and help them to overcome learning burnout by the lecture, group and individual consultation, and special psychological healthy course.

\subsection{Teachers should change the single and bald force-fed teaching mode}

Teachers should emphasize the adopt the heuristic education and the research teaching mode to continually enrich the teaching content, strengthen the charm of teaching process, and make students to enter into the learning and become the master of the learning. Teachers should specially pay attention to the connection between theory and practice, and instruct students to study the knowledge in order to apply it by after-school activities, practice, and probations, enhance their ability to solve problems, and promote them to experience the interests of learning and the value of knowledge in the practice. Teachers should also adopt various and flexible teaching modes to establish the teaching situations, make suspense, and stimulate their curiosity and learning enthusiasm.

\subsection{College students must actively change themselves}

First, college students should emphasize the will training and the self-building of personality. Students should strengthen the endurance to the learning burnout. College students should establish long-range ideas, actively participate in various practices, cultivate their patience and establish the confidence and decision, train their will and quality, and enhance their ability to refuse the entices in the process of overcoming difficulties. At the same time, facing the learning burnout, college students should bravely face the actuality with optimistic attitude, and be good at changing the unreasonable cognitions, and control their emotions by various measures, and keep good learning enthusiasm. They also should self-consciously refuse the influences of bad style and culture in the society, try to cultivate right demands and interests, establish active value view, philosophy, and firm life faith.

Second, college students should establish proper learning target. The so-called "proper" means that the level of the 
target is not too high or too low. Too low learning target can not enhance the level of individual, and too high learning target will be hard to realize, and when the individual is failed, his learning enthusiasm will be struck. Therefore, based on objective analysis of existing knowledge and ability, college students should establish their own learning target which is higher than existing level but can be achieved. Facing thus target, the individual will not flinch, and the realization of the target will also stimulate his enthusiasm of learning to effectively deal with the learning burnout.

Third, college students should properly attribute their failures. Psychologist Julian Bernard Rotter proposed the theory of control point in 1954, and he thought that the interior control was that the result was induced by individual behaviors or decided by individual stable personality; on the contrary, if the thing was induced by the factors except for the individual, it was called as exterior control (Chen, 2007, P.225). Interior controllers are always inclined to attribute their learning failure to the deficient ability and efforts, and their learning motivation is stronger. But exterior controllers are always inclined to attribute their learning failure to the luck, learning task, and other exterior causes, because they thought that these exterior causes make the learning can not be improved and enhanced, and even they attribute the failure to the interior causes, they will only increase too much pressures. Therefore, when facing the learning failure, college students should make objective and exact attributions, and they should not only look for the causes from themselves, but see the influences of objective factors, and find a balance point between exterior causes and interior cause, and form correct cognition to the learning failure, and effectively reduce the level of learning burnout.

Fourth, college students should fully enjoy the happiness of college life. Learning is the main rhythm of the college life, but it is not the whole of the college life, and the learning burnout is a tired state in body and mind when students engage in the learning all along and the happiness in the life could effectively restrain and adjust this bad state. Therefore, students should work and rest in the learning, and after learning, they should actively participate in many recreational activities, develop wide interests, enjoy the happiness and relaxation of the life, and promote their attention to transfer properly, which can effectively loose the body and mind, adjust the nervous state of the brain, and reduce the level of learning burnout.

\section{References}

Chen, Qi \& Liu, Rude. (2007). Contemporary Educational Psychology. Beijing: Beijing Normal University Press, P.225.

Huang, Weiping \& Li, Qian. (2007). Causes and Countermeasures of College Students' Learning Burnout. Studies in Ideological Education, No. 4, P.54.

Li, Weiwei, Chen, Jianzhi \& Yu, Hong. (2007). Condition of Learning Burnout of Sophomores and Juniors and the Relation to Personality Characters. Journal of Jixi University (Comprehensive Edition), 7(5), P.19-21.

Yang, Lixian \& Lian, Rong. (2005). Current Studies and Prospects of Learning Burnout. Journal of Jimei University (Education Science Edition), 8(2), P.54-58.

Zhang, Shu \& Zhang, Pei. (2009). An Analysis of Undergraduates' Learning Burnout in Inner Mongolia. Journal of Baotou Medical College, 25(2), P.140-142. 